\title{
GAIA Small for Gestational Age Level of Diagnostic Certainty
}

National Cancer Institute

\section{Source}

National Cancer Institute. GAIA Small for Gestational Age Level of Diagnostic Certainty. NCI Thesaurus. Code C128674.

A classification of maternal and fetal outcomes relating to small for gestational age, developed by the Global Alignment of Immunization safety Assessment in pregnancy, based on the extent to which the diagnosis has been confirmed. 